\title{
A case report of thin basement membrane nephropathy accompanied by sporadic glomerulocystic kidney disease
}

\author{
Hiroyuki Hashimoto ${ }^{1}$, Naro Ohashi ${ }^{*}$ (D), Naoko Tsuji ${ }^{2}$, Yoshitaka Naito ${ }^{1}$, Shinsuke Isobe ${ }^{1}$, Tomoyuki Fujikura', \\ Takayuki Tsuji ${ }^{1}$, Akihiko Kato ${ }^{2}$, Kandai Nozu ${ }^{3}$, Kazumoto lijima ${ }^{3}$ and Hideo Yasuda ${ }^{1}$
}

\begin{abstract}
Background: Thin basement membrane nephropathy (TBMN) is a relatively common disease. Patients typically present with isolated hematuria, which has a good renal prognosis. In contrast, glomerulocystic kidney disease (GCKD) is a rare disease, associated with slow progressive renal dysfunction. To our knowledge, co-occurring diagnosis of TBMN with GCKD has not been reported previously.

Case presentation: A 30-year old woman was admitted to our hospital for evaluation of hematuria and renal insufficiency. Upon examination, her urinary protein level was $40 \mathrm{mg} /$ day and occult blood in her urine was $2+$. The patient's urinary dysmorphic red blood cell sediment was 30-49/high power field. In contrast, her serum creatinine levels increased from $0.57 \mathrm{mg} / \mathrm{dl}$ to $0.86 \mathrm{mg} / \mathrm{dl}$ during the previous 2-years, without special events. She suffered from far-sightedness and astigmatism beginning at birth; She had no family history of renal disease. Renal biopsy demonstrated cystic dilatation of the Bowman's capsule and atrophy of the glomerular tuft. The glomerular basement membrane (GBM) was thin, with an average thickness of $191 \mathrm{~nm}$. Next-generation sequencing was used to evaluate for mutations in COLAA3 and COLAA4, associated with TBMN, and UMOD, MUC1, and SEC61A1, associated with hereditary GCKD. No pathogenic mutations were identified. We thus diagnosed the patient with TBMN coexistent with sporadic GCKD.

Conclusion: We report the patient diagnosed with TBMN accompanied by sporadic GCKD, based on renal biopsy and genetic testing. Because it is possible that other diseases, such as GCKD, can coexist with TBMN, it is important to consider renal biopsy.
\end{abstract}

Keywords: Thin basement membrane nephropathy, Glomerulocystic kidney disease, Renal biopsy, Genetic testing, Case report

\section{Background}

Thin basement membrane nephropathy (TBMN) is a disease characterized by thinning of the glomerular basement membrane (GBM) mainly caused by gene mutations of type IV collagen $\alpha 3$ or $\alpha 4$ (COL4A3 or COL4A4), which are the component materials of the GBM [1]. Haas et al. established criteria for the lower limit of normal GBM thickness as $230 \mathrm{~nm}$ in male patients and $215 \mathrm{~nm}$ in female patients at the age of 9 -years or older [1].

\footnotetext{
* Correspondence: ohashi-n@hama-med.ac.jp

${ }^{1}$ Internal Medicine 1, Hamamatsu University School of Medicine, 1-20-1

Handayama, Higashi-ku Hamamatsu 431-3192, Japan

Full list of author information is available at the end of the article
}

TBNM is one of the most frequent causes of isolated microscopic hematuria, with an incidence of approximately $1 \%$ in the general population $[2,3]$. In general, urinary protein excretion and blood pressure are normal in patients with TBMN while the characteristic manifestation is persistent or intermittent asymptomatic microscopic hematuria [4].

It is well established that TBMN coexists with other glomerular pathologies. Qazi et al. reported a TBMN prevalence of $47(7.4 \%)$ in a total of 634 biopsies. Among these cases, 17 (36.2\%) had TBMN alone and the remaining 30 (63.8\%) were patients with other glomerular pathologies, as follows: immunoglobulin A (IgA) nephropathy, 9 (19.1\%);

(c) The Author(s). 2019 Open Access This article is distributed under the terms of the Creative Commons Attribution 4.0 International License (http://creativecommons.org/licenses/by/4.0/), which permits unrestricted use, distribution, and reproduction in any medium, provided you give appropriate credit to the original author(s) and the source, provide a link to the Creative Commons license, and indicate if changes were made. The Creative Commons Public Domain Dedication waiver (http://creativecommons.org/publicdomain/zero/1.0/) applies to the data made available in this article, unless otherwise stated. 
focal segmental glomerulosclerosis (FSGS), 9 (19.1\%); pauciimmune crescentic glomerulonephritis, 3 (6.4\%); mesangioproliferative glomerulonephritis, 2 (4.3\%); acute interstitial nephritis, 2 (4.2\%); lupus nephritis, 1 (2.1\%); focal endocapillary proliferative glomerulonephritis, 1 (2.1\%); acute endocapillary glomerulonephritis, 1 (2.1\%), chronic sclerosing glomerulonephritis, 1 (2.1\%), and; both IgA nephropathy and FSGS, 1 (2.1\%) [5].

In contrast, glomerulocystic kidney disease (GCKD), first reported by Ribbert et al. in 1889, is a rare disease in which cystic dilatation of the Bowman's capsule and atrophy of the glomerular tuft are observed. Glomerular cysts are defined as Bowman space dilatation greater than 2 to 3 times the normal size. They occur in disorders of diverse etiology and are associated with a spectrum of clinical manifestations. GCKD is diagnosed in patients with greater than $5 \%$ cystic glomeruli in the kidneys [6].

Lennerz et al. retrospectively assessed 20 cases of GCKD from their records and identified $>230$ cases published in the literature [7]. However, to the best of our knowledge, we believe that this is the first case of TBMN co-occurring with GCKD reported in the literature. Herein, we report on this rare case of a patient with TBMN and sporadic GCKD diagnosed by renal biopsy and genetic testing.

\section{Case presentation}

A 30-year old woman was admitted to our hospital to undergo renal biopsy. According to her medical records, microscopic hematuria was detected on every urine analysis since her birth. At 16-years-old, the potential causes were closely examined. However, no diagnosis was made. Renal biopsy was not performed at that time because the patient's manifestation was only microscopic hematuria without increased urinary protein or other signs of renal dysfunction. A few years later, she chose to stop her regular visits to the doctor. When she was 27-years old, she became pregnant. Upon her initial visit at the department of obstetrics and gynecology, she tested positive for hematuria. In the 30th week of pregnancy, her urine tested positive for the presence of protein but the results returned to normal after puerperium. Further, the patient experienced hypertension soon after delivery, but her blood pressure was normotensive during all other periods. Through entire pregnancy and postpartum, evidence of hematuria remained upon testing her urine samples.

The patient did not experience any subjective or objective symptoms associated with kidney disorders such as, fever, deafness, hemoptysis, or rash. In addition, she was not taking any medication. Her urinary findings did not change over 2-years. The urinary protein level was 60 and $40 \mathrm{mg} /$ day in 2016 and 2018, respectively.
Hematuria was assessed using a urinary occult blood test resulting in 3+ and 2+ in 2016 and 2018, respectively. In addition, the urinary dysmorphic red blood cell (RBC) sediment was 50-99/high power field (HPF) and 30-49/ HPF in 2016 and 2018, respectively. In contrast, serum creatinine levels increased from $0.57 \mathrm{mg} / \mathrm{dl}$ to $0.86 \mathrm{mg} / \mathrm{dl}$ during the same 2-years, without special events.

The patient had a history of far-sightedness, astigmatism, and strabismus from birth as well as polycystic ovarian syndrome diagnosed at the age of 27-years. Her family history included a paternal grandmother with a subarachnoid hemorrhage and a father with hypertension. There was no family history of renal dysfunction or urinary abnormalities.

Physical examination results on admission were as follows: height $152 \mathrm{~cm}$, weight $42.0 \mathrm{~kg}$, body mass index (BMI) $18.2 \mathrm{~kg} / \mathrm{m}^{2}$, body temperature $36.8^{\circ} \mathrm{C}$, blood pressure $99 / 76 \mathrm{mmHg}$, and pulse of 73 beats/min. Hearing loss, skin manifestations, and joint symptoms were not detected. In addition, the patient's respiratory sounds were normal and abdominal tenderness was not detected. Laboratory data showed renal insufficiency [serum creatinine, $0.86 \mathrm{mg} / \mathrm{dl}$; estimated glomerular filtration rate (eGFR), $64 \mathrm{ml} / \mathrm{min} / 1.73 \mathrm{~m}^{2}$ ] and hematuria (urinary occult blood, 3+; urinary dysmorphic RBC sediment, 11.3/HPF) (Table 1). eGFR was calculated by serum creatinine concentrations using the Japanese eGFR equation [8]. The patient's urinary protein level was $60 \mathrm{mg} /$ day, which is classified as normal to mildly increased proteinuria according to the Kidney Disease: Improving Global Outcomes 2012 Clinical Practice Guidelines [9]. Immunological testing showed no abnormal findings (Table 1).

The kidney size was within normal limits (right: $109 \times$ $58 \mathrm{~mm}$; left, $108 \times 62 \mathrm{~mm}$ ) and compression of the left renal vein between the aorta and proximal superior mesenteric artery, which would suggest Nutcracker syndrome, was not detected on abdominal computed tomography $(\mathrm{CT})$. However, the density of the renal cortex was remarkably low (Fig. 1 a). In addition, numerous small high-intensity spots were distributed within the subcapsular cortex on magnetic resonance imaging T2weighted image (Fig. 1 b).

The differential diagnosis of hematuria from early childhood include IgA nephropathy, Alport syndrome, and TBMN. Therefore, we performed renal biopsy the day after admission. Renal histological examination revealed global sclerosis in 5 of 22 glomeruli, cystic dilatation of the Bowman's capsule in 3 of 22 glomeruli (14.1\%), and atrophy of the glomerular tufts (Fig. 2 a and b). Immunofluorescence studies indicated no deposition of immunoglobulins or complement proteins. Type IV collagen staining showed normal expression levels of the $\alpha 5$ chain in the GBM and Bowman's capsule (Fig. $2 \mathrm{c}$ to 
Table 1 Laboratory findings on admission

\begin{tabular}{|c|c|}
\hline Parameter & Value (reference range) \\
\hline \multicolumn{2}{|l|}{ Hematology } \\
\hline WBC count, $/ \mu \mathrm{L}$ & $5630(3000-9000)$ \\
\hline Hemoglobin, g/dL & $10.5(11.0-15.0)$ \\
\hline Platelet count, $10^{4} / \mu \mathrm{L}$ & $21.9(14.5-35.0)$ \\
\hline \multicolumn{2}{|l|}{ Blood chemistry } \\
\hline $\mathrm{sCr}, \mathrm{mg} / \mathrm{dL}$ & $0.86(0.2-0.8)$ \\
\hline $\mathrm{eGFR}, \mathrm{mL} / \mathrm{min} / 1.73 \mathrm{~m}^{2}$ & $64(>90)$ \\
\hline $\mathrm{SUN}, \mathrm{mg} / \mathrm{dL}$ & $13.3(8-22)$ \\
\hline Serum albumin, g/dL & $4.3(3.9-4.9)$ \\
\hline AST, U/L & $16(13-30)$ \\
\hline $\mathrm{ALT}, \mathrm{U} / \mathrm{L}$ & $7(10-40)$ \\
\hline $\mathrm{LDH}, \mathrm{U} / \mathrm{L}$ & $162(110-210)$ \\
\hline $\mathrm{HbA} 1 \mathrm{c}, \%$ & $5.2 \%(4.6-6.2)$ \\
\hline $\mathrm{FBS}, \mathrm{mg} / \mathrm{dl}$ & $93(70-109)$ \\
\hline Triglyceride, mg/dl & $80(50-149)$ \\
\hline T. cholesterol, mg/dl & $166(150-219)$ \\
\hline $\mathrm{HDL}-\mathrm{C}, \mathrm{mg} / \mathrm{dl}$ & $40(40-06)$ \\
\hline FDP-DD, $\mu \mathrm{g} / \mathrm{ml}$ & $<1.0(<1.0)$ \\
\hline PT-INR & $1.00(0.9-1.10)$ \\
\hline \multicolumn{2}{|l|}{ Immunology } \\
\hline $\mathrm{CH} 50, \mathrm{U} / \mathrm{ml}$ & $44(31-49)$ \\
\hline $\mathrm{C} 3, \mathrm{mg} / \mathrm{dL}$ & $77(75-148)$ \\
\hline $\mathrm{C} 4, \mathrm{mg} / \mathrm{dL}$ & $21(14-38)$ \\
\hline ANA & $<40(<40)$ \\
\hline Anti-dsDNA antibody, IU/mL & $<10(<12)$ \\
\hline MPO-ANCA, U/mL & $<1.0(<3.5)$ \\
\hline PR3-ANCA, U/mL & $<1.0(<3.5)$ \\
\hline Rheumatoid factor, IU/mL & $5.2(0-15)$ \\
\hline HIV antibody & Negative \\
\hline HCV antibody & Negative \\
\hline HBV surface antigen & Negative \\
\hline HBV surface antibody & Negative \\
\hline \multicolumn{2}{|l|}{ Urinalysis } \\
\hline Urine occult blood & $2+$ \\
\hline Urine dipstick protein & - \\
\hline Urine $\mathrm{RBC} / \mathrm{HPF}$ & $11.3(0.0-4.9)$ \\
\hline Urine WBC/HPF & $0.7(0.0-5.0)$ \\
\hline Spot urine $P C R, g / g$ & $0.04(<0.15)$ \\
\hline
\end{tabular}

Abbreviations: $A L T$, Alanine aminotransferase, ANA, Antinuclear antibody; ANCA, Antineutrophil cytoplasmic antibody, AST, Aspartate aminotransferase, $\mathrm{CH} 50$, Complement activity, $d s D N A$, Double-stranded DNA, eGFR, Estimated glomerular filtration rate, FBS, Fasting blood sugar, $F D P-D D$, Fibrin degradation products-D, Dimer, HbA1c, Hemoglobin A1c, $H B V$, Hepatitis B virus, $H C V$, Hepatitis C virus, HDL-C, High density lipoprotein cholesterol, HIV, Human immunodeficiency virus, HPF, High power field, INR, International normalized ratio, $L D H$, Lactate dehydrogenase, $M P O$, Myeloperoxidase, $P C R$, Protein-creatinine ratio, $P R 3$, Proteinase 3, $P T$, Prothrombin time, $R B C$, Red blood cell, $S C$ r, Serum creatinine, SUN, Serum urea nitrogen, $T$, Cholesterol total cholesterol, WBC, White blood cell e). The renal biopsy samples obtained for electron microscopy examination did not contain cystic glomeruli and therefore, the detailed appearance of cystic glomeruli could not be evaluated. Electron microscopy revealed thinning of the GBM. The width of the GBM was measured at 30 random points to calculate the average value. The average thickness of the GBM was $191 \mathrm{~nm}$, which exceeded criteria set forth by Haas et al. for the lower limit of normal thickness (215 nm for females) [1] (Fig. $2 \mathrm{f}$ and g). Other morphological abnormalities, including electron-dense deposits and foot process effacement, were not identified on electron microscopy. Based on the above findings, the patient was diagnosed with TBMN accompanied by GCKD.

Although this patient had no recorded family history of GCKD, we examined whether there were any associations with known genetic mutations using next-generation sequencing. There were no pathogenic mutations of $U M O D$, $M U C 1$, or SEC61A1, encoding uromodulin, mucin1, or Sec61 translocon alpha 1 subunit, which are causative proteins of autosomal dominant tubulointerstitial kidney disease, including GCKD. No pathogenic mutations of $C O L 4 A 3$ and $C O L 4 A 4$, encoding type IV collagen $\alpha 3$ and $\alpha 4$ of the GBM, were detected. Based on these results, we diagnosed the patient with TBMN coexistent with sporadic GCKD. The patient was discharged and followed closely without intervention.

\section{Discussion and conclusions}

We diagnosed a patient with TBMN accompanied by sporadic GCKD based on negative genetic tests for commonly associated mutations. The patient presented with isolated hematuria without proteinuria since birth. Urinary protein excretion and blood pressure are typically normal in patients with TBMN and the characteristic manifestation of TBMN is persistent or intermittent asymptomatic microscopic hematuria incidentally discovered on routine urinalysis [4]. Therefore, we suspected the diagnosis of TBMN on admission to the hospital. However, the progressive renal dysfunction in the patient was atypical.

Alport syndrome is a genetically heterogeneous condition characterized by structural abnormalities in the GBM. The clinical course of Alport syndrome is typically manifested as hematuria and progressive renal insufficiency developing from childhood to early- or middleaged adulthood [1]. While our findings reflected those of Alport syndrome, the absence of diseased proteinuria, anterior lenticonus, and hearing loss did not correspond with that diagnosis. IgA nephropathy is the most common glomerulonephritis globally, with a clinical presentation of asymptomatic hematuria. Isolated microscopic hematuria with minimal proteinuria is regarded as a favorable prognosis [10]. However, one factor associated 


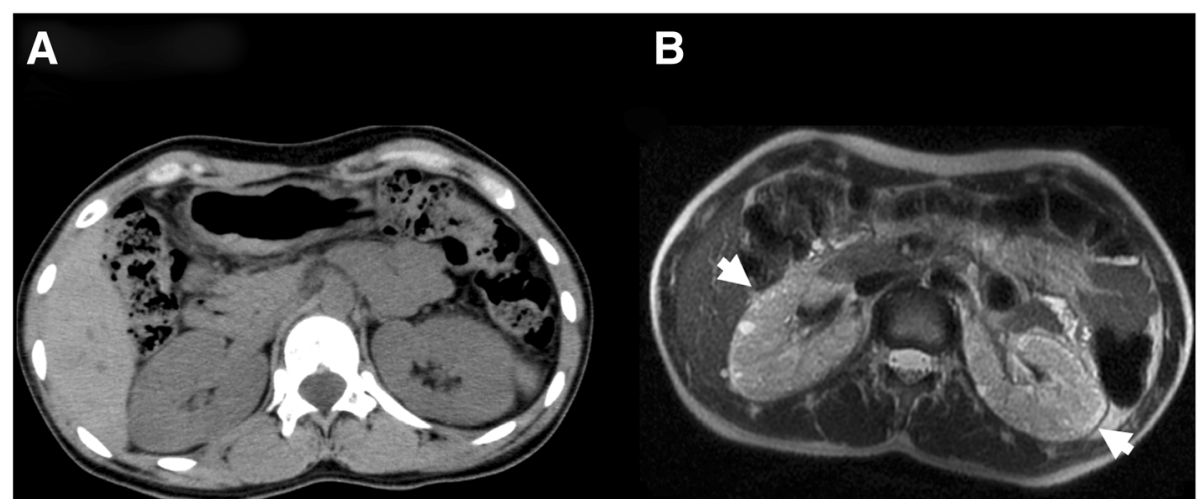

Fig. 1 Kidney morphology. a Computed tomography scan of the kidney. The density of the renal cortex was remarkably low in the bilateral, normal sized kidneys. b Magnetic resonance imaging (MRI) of the kidneys. Numerous small high intensity spots (arrow) were distributed within the subcapsular cortex on T2-weighted MRI

with poor prognosis is proteinuria [11]. In this case, the levels of proteinuria remained normal to mildly increased, despite aggravated renal function. Therefore, it was difficult to confirm IgA nephropathy as a significant differential diagnosis. Hence, we performed a renal biopsy. As a result, we recorded no longitudinal splitting of the lamina densa of the GBM and Alport syndrome was ruled out. In addition, mesangial cell proliferation and mesangial matrix accumulation were not visible, and immunofluorescence studies indicated no deposition of IgA. Therefore, IgA nephropathy was also ruled out.
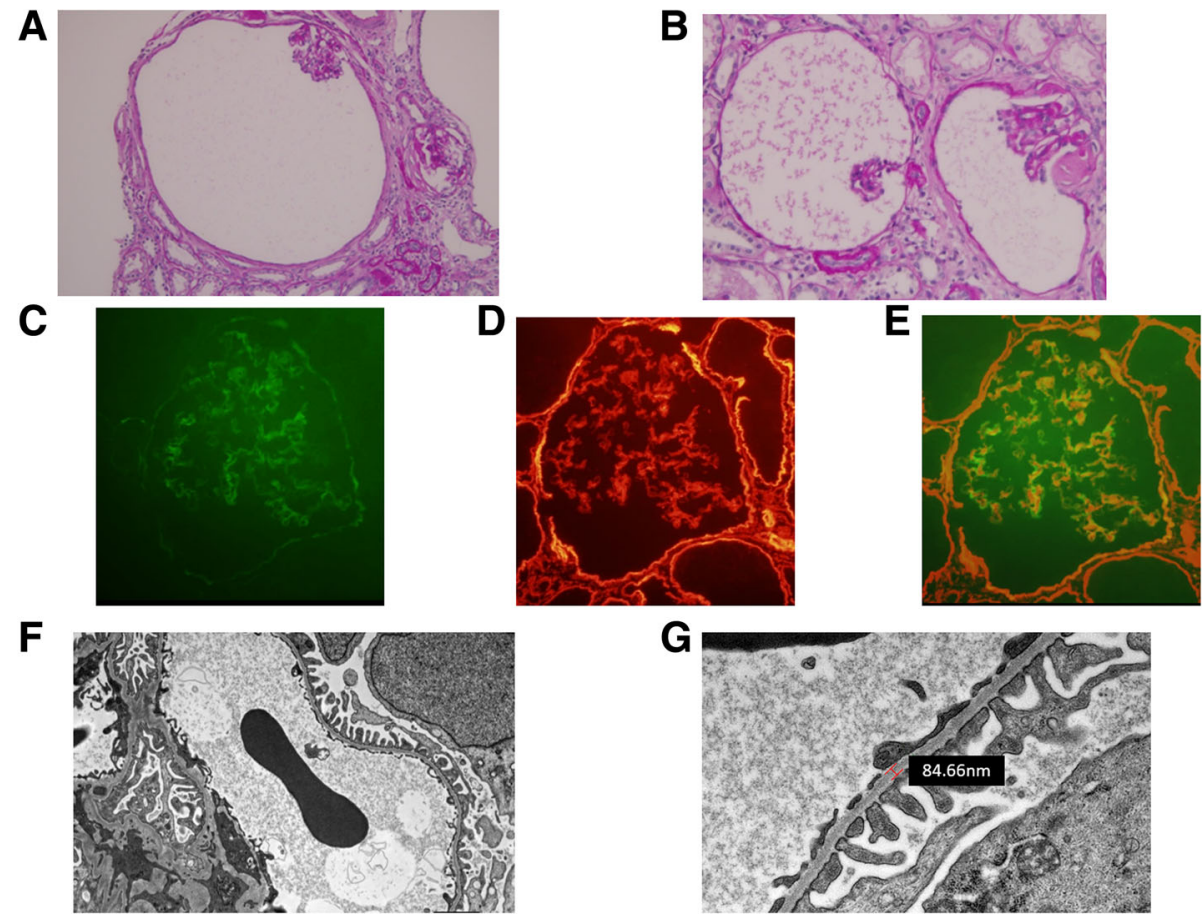

Fig. 2 Renal biopsy findings. Light microscopy. The glomerulus shows cystic dilatation of Bowman's capsule and atrophy of the glomerular tuft, $(1 \mathrm{~A})$ (periodic acid-Schiff (PAS) stain: original magnification $\times 100$ ) and $(1 B)(P A S$ stain: original magnification $\times 400)$. Type IV collagen staining. Immunofluorescence study was performed to evaluate the expression levels of type IV collagen. FITC-conjugated-anti a5 chain is observed in the glomerular basement membrane, part of the tubular basement membrane, and Bowman's capsular basement membrane (1C). In addition, Texas Red-anti a2 chain revealed the renal basement membrane structure (1D). Basement membranes where two fluorescence were present appear green to yellow (1E) (original magnification $\times 400$ ). Fluorochrome-conjugated anti collagen IV cocktail for Alport's syndrome was used (Cosmo Bio Ltd. Co., Tokyo, Japan). Electron microscopy. Morphological abnormalities, apart from thinning of the glomerular basement membrane, were not found; there were no electron dense deposits ( 1 F; original magnification $\times 3000$ and $1 \mathrm{G}$; original magnification $\times 10,000$ ) 
Angiotensin converting enzyme inhibitors or angiotensin receptor blockers are recommended to prolong renal survival for patients of Alport syndrome or TBMN with or without proteinuria. Although she had no known genetic mutation associated with Alport syndrome or TBMN, angiotensin converting enzyme inhibitors or angiotensin receptor blockers might be her potential option [12-14].

The clinical manifestations of GCKD are divided into early-onset (in neonates) and late-onset (in adults). Renal function in early-onset GCKD follows a stable course for several years and may progress to end-stage renal disease in $\geq 3$-years. Late-onset GCKD typically shows less severe renal impairment [7]. It is possible that superimposed glomerulonephritis [15] or distinct diseases [16] can explain the different clinical manifestations of GCKD.

We were able to diagnose TBMN coexistent with GCKD after reviewing the renal biopsy specimen in this case. This illustrates the importance of renal biopsy when clinical findings do not correlate well with the expected clinical course of renal disease. Since it is possible to treat IgA nephropathy following diagnosis via renal biopsy, the biopsy in this case was meaningful, allowing the patient to be discharged and followed closely without intervention. Therefore, we believe renal biopsy was necessary to define the appropriate treatment or close follow-up.

Because GCKD is a rare disease and $80 \%$ cases of GCKD occur in early life, nephrologists seldom encounter adult patients with GCKD, making it difficult to come up with the diagnosis of GCKD. GCKD was divided into 5 categories by Lennerz et al., according to the etiology and spectrum of clinical manifestations, as follows: category I, GCKD in polycystic kidney disease; category II, hereditary GCKD; category III, syndromic GCKD; category IV, obstructive GCKD; category V, sporadic GCKD [7]. Among the 5 categories, GCKD in polycystic kidney disease and hereditary GCKD were ruled out in this patient due to the absence of cysts in other organs and absence of a family history, respectively. Obstructive GCKD was ruled out due to lack of urinary tract obstruction. Finally, syndromic GCKD was ruled out due to the absence of associated findings, such as hypopigmented macules, angiofibroma, and shagreen patches, which are indicative of tuberous sclerosis [17], and high forehead, epicanthal folds, hypoplasia of supraorbital ridges, and midface, which are indicative of Zellweger syndrome [18]. Therefore, this patient was suspected to have sporadic GCKD. However, there was no history of the primary causes of sporadic GCKD including ischemic damage, such as progressive systemic sclerosis [19], hemolytic uremic syndrome [20], or exposure to drugs [7], such as lithium.
Most subjective and objective symptoms of syndromic or inherited GCKD occur from early childhood. For example, tuberous sclerosis is the most frequent syndromic GCKD, which is found in early childhood. Zellweger syndrome, which is primarily associated with syndromic GCKD, occurs at birth. In addition, inherited GCKD, which is caused by uromodulin disorders, is typically diagnosed in school-age children [21]. However, familiar hypoplastic GCKD, which is caused by mutations in the gene encoding hepatocyte nuclear factor- $1 \beta$, can occur from fetal stage to the age of 39-years [22]. Results from previous reports indicated that our patient may not express abnormal phenotypes associated with syndromic or inherited diagnosis, even at the age of 30-years. Therefore, we performed genetic testing for $U M O D$, $M U C 1$, and SEC61A1, encoding uromodulin, mucin1, and Sec61 translocon alpha 1 subunit. Because no abnormal genetic mutations were detected in this case, we diagnosed the patient with sporadic GCKD.

It is possible to detect any gene mutation in the patient by analyzing the full genome sequence. However, even if we can detect the gene mutations, it is impossible to determine whether the detected gene mutations are responsible for GCKD since the particular gene location has not been identified. The examination of full genome sequences from several families that include individuals with and without GCKD will enable us to determine the mutation responsible for GCKD. Therefore, it is not possible to provide the definitive diagnosis at this time.

Additionally, no mutations of COLAA3 and COL4A4 were identified. However, this result is not necessarily incompatible with the diagnosis of TBMN, because mutations of COLAA3 and COL4A4 are associated with only $40 \%$ of TBMN cases. Thus, many as-yet unknown genes are also responsible for thinning of the GBM [23, 24].

The genetic testing was negative and did not change the outcome or affect treatment in the present case. However, some important information was obtained. First, negative results for commonly associated mutations on the genetic test led to a diagnosis of sporadic GCKD. Second, no known mutations associated with GCKD or TBMN were detected in the present case by genetic testing; therefore, the causal relationship between GCKD and TBMN remains unclear. However, it is possible that both GCKD and TBMN are caused by a single unrecognized mutation. The fact that there was no family history coincides with this hypothesis.

There are other diseases associated with cystic lesions and basement membrane abnormalities, such as hereditary angiopathy with nephropathy, aneurysms, and muscle cramps (HANAC) syndrome. This syndrome is an autosomal dominant syndrome caused by mutations in COL4A1, which encodes the $\alpha 1$ chain of collagen IV, a major component of the basement membrane. In this 
syndrome, renal disease manifests in multicystic kidneys, hematuria in some cases, and decreased eGFR. In addition, renal morphology can indicate basement membrane abnormalities [25, 26]. These features are similar to TBMN accompanied by GCKD, as reported in our case. However, multicystic kidneys associated with renal cysts, not glomerular cysts, and morphological changes in the tubular, vascular, and Bowman's capsule basement membrane, but not glomerular basement membrane, are found in HANAC syndrome. This is because the $\alpha 1$ chain is a component of tubular, vascular, and Bowman's capsule basement membranes, but not glomerular basement membranes. Therefore, we consider our case different from HANAC syndrome.

In conclusion, we report the case of TBMN accompanied by sporadic GCKD. TBMN is one of the most frequent causes of isolated microscopic hematuria with no associated physical findings and normal laboratory findings, except hematuria. However, because it is possible that other diseases, such as GCKD, coexist with TBMN, it is important to consider renal biopsy, in addition to conducting serum serological and urine studies. Moreover, because GCKD has 5 categories and multiple etiologies, it is possible that the patient does not yet express the associated abnormal phenotype at the time of diagnosis. Therefore, it is also important to consider genetic testing.

\section{Abbreviations \\ BMI: Body mass index; CT: Computed tomography; eGFR: Estimated glomerular filtration rate; FSGS: Focal segmental glomerulosclerosis; GBM: Glomerular basement membrane; GCKD: Glomerulocystic kidney disease; HANAC: Hereditary angiopathy with nephropathy, aneurysms, and muscle cramps; HPF: High power field; IgA: Immunoglobulin A; RBC: Red blood cell; TBMN: Thin basement membrane nephropathy}

\section{Acknowledgements}

None.

\section{Authors' contributions}

$\mathrm{HH}, \mathrm{NO}, \mathrm{NT}, \mathrm{YN}, \mathrm{SI}, \mathrm{TF}, \mathrm{T}, \mathrm{AK}$ and $\mathrm{HY}$ treated the patient. NT took the pictures of electron microscopy and measured the thickness of basement membrane. KN and Kl performed genetic testing and provided genomic opinions. $\mathrm{HH}$ wrote the first draft. $\mathrm{NO}$ and $\mathrm{HY}$ wrote the final draft. All authors reviewed and approved the final version of this manuscript.

\section{Funding}

The authors have no relevant financial interests to disclose.

\section{Availability of data and materials}

All data generated during this study are included in this published article.

\section{Ethics approval and consent to participate}

Written informed consents were obtained from the patient for ethics approval and consent, because we examined known genetic mutations using next-generation sequencing, even though the treatments were not administered for research purposes. The ethics committee of Hamamatsu University School of Medicine approved the committee's reference number (17-032).

\section{Consent for publication}

Written informed consent was obtained from the patient for publication of this case report and any accompanying images. A copy of the written consent is available for review by the Editor of this journal.

\section{Competing interests}

The authors declare that they have no competing interests.

\section{Author details}

${ }^{1}$ Internal Medicine 1, Hamamatsu University School of Medicine, 1-20-1 Handayama, Higashi-ku Hamamatsu 431-3192, Japan. ²Blood Purification Unit, Hamamatsu University School of Medicine, 1-20-1 Handayama, Higashi-ku Hamamatsu 431-3192, Japan. ${ }^{3}$ Department of Pediatrics, Kobe University Graduate School of Medicine, 7-5-1 Kusunoki-cho, Chuo-ku, Kobe 650-0017, Japan

Received: 29 November 2018 Accepted: 3 July 2019

Published online: 09 July 2019

\section{References}

1. Haas M. Alport syndrome and thin glomerular basement membrane nephropathy: a practical approach to diagnosis. Arch Pathol Lab Med. 2009; 133:224-32

2. Haas M. Thin glomerular basement membrane nephropathy: incidence in 3471 consecutive renal biopsies examined by electron microscopy. Arch Pathol Lab Med. 2006;130:699-706.

3. Kashtan CE. Alport syndrome and thin basement membrane nephropathy: diseases arising from mutations in type IV collagen. Saudi J Kidney Dis Transpl. 2003:14:276-89.

4. Tryggvason K, Patrakka J. Thin basement membrane nephropathy. J Am Soc Nephrol. 2006;17:813-22.

5. Qazi RA, Bastani B. Co-existence of thin basement membrane nephropathy with other glomerular pathologies; a single center experience. J Nephropathol. 2015;4:43-7.

6. Bernstein J, Landing BH. Glomerulocystic kidney disease. Prog Clin Biol Res. 1989:305:27-43

7. Lennerz JK, Spence DC, Iskandar SS, Dehner LP, Liapis H. Glomerulocystic kidney: one hundred-year perspective. Arch Pathol Lab Med. 2010;134:583605.

8. Matsuo S, Imai E, Horio M, Yasuda Y, Tomita K, Nitta K, et al. Collaborators developing the Japanese equation for estimated GFR. Revised equations for estimated GFR from serum creatinine in Japan. Am J Kidney Dis. 2009;53: 982-92.

9. Levey AS, de Jong PE, Coresh J, El Nahas M, Astor BC, Matsushita K, et al. The definition, classification, and prognosis of chronic kidney disease: a KDIGO controversies conference report. Kidney Int. 2011:80:17-28.

10. Gutiérrez E, Zamora I, Ballarín JA, Arce Y, Jiménez S, Quereda C, et al. Grupo de Estudio de Enfermedades Glomerulares de la Sociedad Española de Nefrología (GLOSEN). Long-term outcomes of IgA nephropathy presenting with minimal or no proteinuria. J Am Soc Nephrol. 2012;23:1753-60.

11. Barbour SJ, Reich HN. Risk stratification of patients with IgA nephropathy. Am J Kidney Dis. 2012;59:865-73.

12. Savva I, Pierides A, Deltas C. RAAS inhibition and the course of Alport syndrome. Pharmacol Res. 2016;107:205-10.

13. Palmer SC, Mavridis D, Naarese E, Craig JC, Tonelli M, Salanti G, et al. Comparative efficacy and safety of blood pressure-lowering agents in adults with diabetes and kidney disease: a network meta-analysis. Lancet. 2015; 385(9982):2047-56.

14. Zhang Y, Wang F, Ding J, Zhang H, Liu X, Wang S, et al. Long-term treatment by ACE inhibitors and angiotensin receptor blockers in children with Alport syndrome. Pediatr Nephrol. 2016;31(1):67-72.

15. Oh Y, Onoyama K, Kobayashi K, Nanishi F, Mitsuoka W, Ohchi N, et al. Glomerulocystic kidneys. Report of an adult case. Nephron. 1986;43:299-302.

16. Bernstein J. Glomerulocystic kidney disease--nosological considerations. Pediatr Nephrol. 1993;7:464-70.

17. Webb DW, Clarke A, Fryer A, Osborne JP. The cutaneous features of tuberous sclerosis: a population study. Br J Dermatol. 1996;135:1-5.

18. Percy AK, Rutledge SL. Adrenoleukodystrophy and related disorders. Ment Retard Dev Disabil Res Rev. 2001;7:179-89. 
19. Sessa A, Giordano F, Meroni M, Battini G, Torri-Tarelli L, Volpi A. Glomerulocystic kidney in a patient affected with progressive systemic sclerosis. Nephron. 1988;48:173-4.

20. Emma F, Muda AO, Rinaldi S, Boldrini R, Bosman C, Rizzoni G. Acquired glomerulocystic kidney disease following hemolytic uremic syndrome. Pediatr Nephrol. 2001;16:557-60.

21. Rampoldi L, Caridi G, Santon D, Boaretto F, Bernascone I, Lamorte G, et al. Allelism of MCKD, FJHN and GCKD caused by impairment of uromodulin export dynamics. Hum Mol Genet. 2003;12:3369-84.

22. Bingham C, Bulman MP, Ellard S, Allen LI, Lipkin GW, Hoff WG, et al. Mutations in the hepatocyte nuclear factor-1 beta gene are associated with familial hypoplastic glomerulocystic kidney disease. Am J Hum Genet. 2001; 68:219-24.

23. Fallerini C, Dosa L, Tita R, Del Prete D, Feriozzi S, Gai G, et al. Unbiased next generation sequencing analysis confirms the existence of autosomal dominant Alport syndrome in a relevant fraction of cases. Clin Genet. 2014; 86:252-7.

24. Papachariou L, Demosthenous P, Pieri M, Papazachariou L, Demosthenous $\mathrm{P}$, Pieri $\mathrm{M}$, et al. Frequency of COL4A3/COL4A4 mutations amongst families segregating glomerular microscopic hematuria and evidence for activation of the unfolded protein response. Focal and segmental glomerulosclerosis is a frequent development during ageing. PLoS One. 2014:9(12):e115015.

25. Plaisier E, Alamowitch S, Gribouval O, Mougenot B, Gaudric A, Antignac C, et al. Autosomal-dominant familial hematuria with retinal arteriolar tortuosity and contractures: a novel syndrome. Kidney Int. 2005;67:2354-60.

26. Plaisier E, Gribouval O, Alamowitch S, Mougenot B, Prost C, Verpont MC, et al. COL4A1 mutations and hereditary angiopathy, nephropathy, aneurysms, and muscle cramps. N Engl J Med. 2007;357:2687-95.

\section{Publisher's Note}

Springer Nature remains neutral with regard to jurisdictional claims in published maps and institutional affiliations.

Ready to submit your research? Choose BMC and benefit from:

- fast, convenient online submission

- thorough peer review by experienced researchers in your field

- rapid publication on acceptance

- support for research data, including large and complex data types

- gold Open Access which fosters wider collaboration and increased citations

- maximum visibility for your research: over $100 \mathrm{M}$ website views per year

At $\mathrm{BMC}$, research is always in progress.

Learn more biomedcentral.com/submissions 